\title{
Transcranial Magnetic Stimulation and the Understanding of Behavior
}

David Pitcher ${ }^{1}$ and Vincent Walsh ${ }^{2}$

\author{
1. Dept of Psychology \\ University of York \\ Heslington \\ York, Y010 5DD \\ UK \\ david.pitcher@york.ac.uk \\ 2. Institute of Cognitive Neuroscience \\ University College London \\ 17 Queen Sq. \\ London WC1N 3AR \\ UK \\ v.walsh@ucl.ac.uk
}




\section{Abstract}

The development of the use of Transcranial Magnetic Stimulation in the study of psychological functions has entered a new phase of sophistication, largely due to increasing physiological knowledge of its effects, and using it in combination with other techniques. This article presents the current state of our understanding of the mechanisms of TMS in the context of designing and interpreting psychological experiments. We discuss the major conceptual advances in behavioral studies using TMS, which are almost entirely a consequence of the physiological and technical achievements, and the contributions they have made to the understanding of perceptual and cognitive processes. In doing so we summarize the different uses and challenges of TMS in mental chronometry, perception, awareness, learning and memory.

Key Words: Transcranial Magnetic Stimulation (TMS); State-Dependency; Spike timing-dependent plasticity (STDP); TMS-imaging; Single unit recording. 


\section{Contents}

Introduction 680

The Five Big Factors in the New-Era of TMS and Psychology 331

Physiology and Behavior 1221

Anatomical Networks 1116

Spike Timing-Dependent Plasticity 1663

State-Dependency 808

Behavioral Findings 1334

Conclusion 295 


\section{Introduction}

The ability to non-invasively stimulate the human brain has many important implications for psychology and cognitive neuroscience. In the first wave of human transcranial magnetic brain stimulation (TMS) studies of behavior there was a disconnection between attempts to produce neuropsychological lesion-like effects, and our understanding of the fundamental physiology of the method. The drive to understand the physiology was led by studies of motor cortex because of the reliability and accessibility of the motor evoked potential (MEP) (Ziemann, 2008). As psychologists trying to mimic effects seen in neuropsychological patients or test theories about the timing or sequences of processes, we had to try to translate our understanding of the excitatory and inhibitory effects of TMS over M1 into the language of our own studies. Not easy. If we reproduced a clinical effect such as, say, language deficits (Devlin \& Watkins, 2007), spatial neglect (Bjoertomt et al., 2002; Fu et al., 2017;), impaired attention (Ellison et al., 2007), or dyscalculia (Cohen Kadosh et al., 2007; Göbel et al., 2006), had we shown that the stimulated area was the key causal area for a behavior, had we inadvertently stimulated a neighbouring region due to current spread, or had we secondarily stimulated another, distal, anatomically connected region? (see Bestmann et al., 2008; Miniussi et al., 2013; Polanía et al., 2018; Romei et al., 2016b; Siebner et al., 2009). There were good grounds for making inferences about anatomical and physiological effects based on experimental controls for stimulation site, chronometry, and task specificity, and many of the findings were interesting and had face validity in a neuropsychological sense, but explaining the effects in any neural sense was more than merely difficult.

The problem was that most areas outside of M1 are "silent" in the sense that they do not give a measurable output comparable with MEP. One might call this first wave of TMS studies in cognition (say, between 1987 and 2007/8) the "point and shoot era." There were many interesting and replicable effects that remain so despite the limitations of the time, and they have been reviewed many times (e.g. de Graaf et al., 2014; Parkin et al., 2015; Walsh \& Pascual-Leone, 2003), but the absence of credible mechanistic explanations limited the impact and slowed our progress.

To imbue behavioral TMS findings with physiological credence three mechanisms were postulated. The first was that the effects of TMS on cognitive functions were caused by "neural noise" (Miniussi et al., 2013; Walsh \& Cowey, 2000) whereby TMS induced disorganised activity in the region stimulated preventing it from carrying out its normal function. A competing concept (Harris et al., 2008) was that TMS suppressed neural activity - decreasing the signal rather than adding noise. To account for behavioral enhancements as well as impairments caused by TMS, Schwarzkopf et al (2011) proposed that TMS induced stochastic resonance effects such that at 
lower intensities it could facilitate stimulus detection. It was clear, however, that without direct recording experiments these proposals remained untested hypotheses.

There are many high quality introductions and reviews of the history, physics, importance of coil orientation, differences between online and offline stimulation, and of early studies of cognition, that we recommend for these aspects of TMS (Hoogendam et al., 2010; Parkin et al., 2015; Peterchev et al., 2012; Walsh \& Pascual-Leone, 2003; Wasserman et al., 2008). We focus here on taking a perspective over the last 10-12 years, during which time there have been important technical, conceptual, and empirical advances. We address only TMS because the volume, intellectual substance, technical accomplishment, and psychological significance of the work over this period are immense and deserve a single treatment. Advances have also been made in other areas of non-invasive human brain stimulation (transcranial direct current stimulation; transcranial alternating current stimulation; transcranial random noise stimulation; low-intensity focussed ultrasound stimulation), all of which deserve a separate treatment (see Antal \& Hermann, 2016; Buch et al., 2017; Chrysikou et al., 2017; Fertonani \& Miniussi, 2017; Horvath et al., 2015; Parkin et al., 2015; Polanía et al., 2018; Schroeder et al., 2017; Reed \& Cohen Kadosh, 2018).

\section{The Five Big Factors in the New-Era of TMS and Psychology}

The maturation of the field is based on five pillars of progress. The first of these is the link between physiological mechanisms and behavioral changes caused by TMS. We will highlight recent work single unit recording from non-human primates. The second pillar is the use of TMS with brain imaging to understanding and human cognitive networks. The third pillar is the adoption and development of paired associative stimulation (PAS) from studies of spike timingdependent plasticity in the motor system (Hebb, 1949; Müller-Dahlhaus et al., 2010; Stefan et al, 2000, 2002; Wolters et al., 2003), which has led to inventive developments in dual coil corticocortical PAS (ccPAS) in cognitive studies. The fourth pillar is State-Dependency, which places constraint on the hypotheses, execution, and interpretation of TMS experiments in cognition, and has become a central concept in design and interpretation in TMS studies in physiology (Pasley et al., 2009), ccPAS (Santarnecchi, 2018), TMS-fMRI (Johnen et al., 2015), TMS-EEG (Herring et al., 2015), and behavior (Mazzoni et al., 2017). The final pillar is that of behavior: at the end of the day, psychologists use TMS to improve our understanding of behavior and to test ideas about cognitive functions. Here too there has been a lot of progress since the "point and shoot" era. Some surprising findings have emerged in memory (van Lamsweerde \& Johnson, 2017; Rose et al., 2016, Wang et al., 2014; Zokaei et al., 2014), visual and motor selection and eye tracking (Bestmann \& Duque, 2016; 
Vesia et al., 2010; 2019), and awareness (e.g. Allen et al., 2014; de Graaf ef al., 2014; Rounis et al., 2010). Of course none of these pillars stand in isolation, and as methods and designs improve, as we will see below, experiments and new findings increasingly stand on several pillars simultaneously. The work we cover here represents real progress, and we hope the overview and characterisation of the field will provide a framework to help the reader understand their significance, and to make their own contributions.

\section{Physiology and Behavior}

The questions received most often when presenting TMS findings are, "how do you know what it's doing?" How far does it spread? What other parts of a network does it interfere with? What is happening at the neuronal level? How long do the effects last? If we consider a single pulse of TMS we can now begin to give good responses to these concerns.

The question of spread was always an inferential one, based on the observation that MEPs or behavioral effects would disappear if the coil was moved by a few millimetres or rotated to a different angle (see Walsh \& Pascual-Leone, 2003; Wasserman et al., 2008). Modelling the physiological spread of TMS was difficult because CSF, and white/grey matter have different conductivities, modelling secondary effects is challenging and of course the models don't address behavioural states (Aberra, et al, 2018; 2020).

The approach needed to illuminate the mechanistic questions was direct physiological recordings. Initially these were carried out on anaesthetised preparations (Allen et al., 2007; de Labra et al., 2007; Moliadze et al., 2003; Pasley et al., 2009). From these studies we learned that TMS had specific excitatory or inhibitory effects in two phases (inhibition followed by a longer period of excitation); that the effects of paired pulse TMS in M1 was mirrored in evoked potential activity in visual cortex (Moliadze et al., 2008), and that TMS-evoked potential (TEP) activity was "state-dependent" (i.e. related to level of neuronal activity immediately prior to stimulation) (Pasley et al., 2009). All this pointed in the right direction with regard to behavioral TMS findings but, as was discovered in the 1960s and 1970s era of single unit recording in the visual cortex, "the cortex dissolves in anaesthesia" (Gross, 1998, p 197). To make the link between physiology and behavior required recordings from awake, behaving monkeys. Ortuno et al., (2014) showed two interesting effects when stimulating V1 and recording from the LGN in monkeys performing a visual detection task. The TMS-induced LGN changes in activity were direct evidence of organised activity at a secondary location, and the effects were state-dependent. From a psychological point of view it was of particular interest that these effects were induced using a paradigm developed in 
human studies ( $\sim 1 \mathrm{~Hz}$ offline - Gangitano et al.,2002). Meuller et al., (2014) then developed the methods that allowed recording from single units in the area of stimulation.

The most comprehensive and recent advance of relevance to psychologists is from single unit recordings in non-human primates trained on a visually-cued reach, grasp, lift, and hold task Romero et al., 2019a). On some trials the monkeys received single pulse TMS over PFG at either 60 or $120 \%$ of motor threshold. Recordings were taken from over 500 cells in area PFG of the inferior parietal cortex contralateral to the hand used in the task, and analysed as a function of TMS intensity, distance from the stimulating coil, and task-relevance. At $120 \%$ of MT there were clear effects of TMS on single units. The TMS pulse induced an artefact of course, so the experiment analysed activity from $10 \mathrm{~ms}$ after the pulse. The main effect of TMS was to induce a volley of action potentials during the first $50 \mathrm{~ms}$. In neurons that were not task-related this occurred irrespective of the stage of the task (cue, lift, grasp, hold) at which TMS was delivered. In other units the early excitation was followed by a period of inhibition, and a third phase of excitation that could last as long as $250 \mathrm{~ms}$ in a small number of neurons (population average $<100 \mathrm{~ms}$ ). Analysis of the distance from the coil and comparisons of neighbouring neurons showed that TMS effects were limited to as little as $2-3 \mathrm{~mm}$. At $60 \%$ of motor threshold TMS had almost no physiological effects.

The final physiological gift from this experiment was the finding that single pulse TMS also induced an increase in low-frequency oscillations both in the centre and periphery of the stimulated regions. These oscillations, mostly in the delta and lower theta ranges are strong candidates for the generations of evoked potentials and long range, cortico-cortical effects of TMS. The oscillations are less salient than those elicited by TMS in EEG studies (Bergmann et al., 2016; Fecchio et al., 2017; Herring et al., 2015; Rogasch et al., 2015; Rosanova et al., 2009; Thut et al., 2017; Thut \& Miniussi, 2009). But as Romero et al., point out, single unit responses and EEG measures are at different spatial and temporal scales. The relationship between the relatively local, low frequency oscillations at the single unit level and the global and more varied frequencies in EEG and MEG is a clear direction for future research.

\section{Figure 1 about here}

These physiological results are impressive, but from a psychological point of view perhaps most interesting is the finding that in some neurons the transient burst in the first phase of the recordings and the reduced activity in the second phase were paralleled with performance on the task. Neurons in PFG are known to be involved in action goal-selection (Bonini et al, 2011; Rozzi et al., 2008) and grasping behavior (Davare et al., 2010Nelissen \& Vanduffel, 2011). This is the 
strongest link to date directly associating TMS-induced neural activity changes to an effect on behavior.

Romero et al., (2019b) have also begun to assess the effects of continuous theta burst stimulation (CTBS) on single unit responses. They measured neural responses after 300 pulses of cTBS and found that the large majority of neurons in area PFG showed significant reduction in activity. The responses fell into three groups: an early group in which $47 \%$ of cells were affected in the first 5 mins after cTBS; a later group in which 44\% showed inhibitory effects between 5 and 30 minutes; and a very late group of remaining cells that showed reduced excitability later than 30 minutes post cTBS. These results show that theta burst stimulation, although based on animal studies and used widely in behavioral studies, does not have unitary effects, and this must now be taken into consideration in interpreting behavioral studies (e.g. Huang et al., 2005; Rahnev et al., 2013; Rounis et al., 2010).

These findings are a landmark in understanding the physiological effects of TMS and, at the very least, provide a more than respectable starting point in answering questions about local spread, interaction with task relevant neurons, and duration of effects. They also help to settle the muddle of metaphors between neural noise, signal reduction, and stochastic resonance accounts of TMS effects. It seems we were all right, which means we were all also wrong: Nobody won and no one must claim prizes. The early and late excitatory responses (Fig 1) correspond with a large noise-like effect the neurons would otherwise not have produced. The second phase of reduced responses is exactly what the signal reduction hypothesis predicted. The oscillatory phase, unlike the earlier burst and inhibition phases, occurred with sub-threshold stimulation, and this is consistent with the stochastic resonance account. But rather than defend one's own partly-correct metaphor, this tri-temporal pattern of single unit responses forces a rethink about how we interpet behavioural results.

The question of what other parts of a network are affected by TMS is the subject of the next section.

\section{Anatomical Networks}

Combining TMS with fMRI enables us to map the remote effects of targeted neural disruption across the entire brain. The value of this approach is threefold. Firstly, it allows us to map the secondary effects of TMS. Secondly, any complex human behavior is computed across a network of areas distributed across the brain. Experiments that combine TMS with fMRI can causally map the 
extent and functionality of these networks (Bestmann et al., 2005, 2008b, 2010; Blankenburg et al., 2008; Lee \& D’Esposito, 2012; Pitcher et al., 2014; Ruff et al., 2006, 2009; Siebner et al., 2009). Thirdly, there are cortical and sub-cortical areas that cannot be directly stimulated by TMS. However, recent combined TMS /fMRI studies have demonstrated that stimulating lateral brain areas can modulate the neural response in remotes areas including the parahippocampal gyrus (Mullin et al., 2013), hippocampus (Wang et al., 2014; Warren et al., 2019) and amygdala (Pitcher et al., 2017). Many compelling studies have now combined TMS and neuroimaging to exploit these advantages.

One research area in which combined TMS-fMRI studies have proven especially useful is in the study of how frontal cortex exerts top-down control over visual perception and decision making. Following from stimulation studies that had shown top-down control of frontal cortex in visual discrimination tasks (Silvanto et al., 2006), Ruff and colleagues recorded fMRI data while TMS was delivered over the frontal eye field (FEF). They revealed that stimulation of the right FEF reduced activity of the retinotopic representation of the central visual field in areas V1 through V4, while also increasing the representations of the peripheral visual field. A follow-up TMS study confirmed the behavioral relevance of these results by demonstrating that TMS delivered over the FEF enhanced perceived contrast for peripheral relative to central visual stimuli. This landmark study demonstrates how combining TMS with fMRI goes beyond what could have been accomplished using each method alone.

Combined TMS-fMRI studies have proved invaluable in studying other cognitive networks. Many single site studies have addressed the causal role of an area in various psychological functions (Dayan et al., 2013; de Graaf et al., 2014; Parkin et al., 2015; Pitcher et al., 2007), but TMSfMRI has allows us to map the remote effects of neural disruption on the brain networks. This is a move from aerial causality to inter-aerial causality: the previously troubling question of whether behavioural effects were due to secondary stimulation is now a tractable question of interest. A good example is the face perception network. Imaging studies show that different brain areas preferentially represent different facial aspects such as emotion, identity, attentional focus, and social cues but is limited in what they can say about how these areas functionally interact. TMSfMRI can systematically disrupt these brain areas and examine the causality of connections in network. Pitcher et al., (2014) used this approach to distinguish the cortical pathways that process dynamic and static features of faces. Theta-burst TMS (TBS) was delivered over the right occipital face area (OFA) or the right posterior superior temporal sulcus (rpSTS). Contrary to existing face processing models (Haxby et al., 2000), invariant facial aspects, like identity, and changeable facial 
aspects, such as expression, were found to be processed via dissociable cortical pathways that begin in early visual cortex.

While face areas like the OFA and pSTS can be directly stimulated (Pitcher et al., 2007; Pitcher, 2014) other parts of the face network, such as the amygdala, cannot be directly targeted by TMS but can be accessed by network analysis of secondary stimulation. Neuroanatomical studies have identified a cortical pathway from the superior temporal sulcus (STS) into the amygdala (Aggleton et al., 1980). Pitcher et al. (2017) combined offline TBS with fMRI to test whether they could identify causal evidence for this cortical pathway in the human brain. Participants were scanned while viewing face and object videos. TBS delivered over the rpSTS prior to fMRI reduced the BOLD response specific to faces in the rpSTS, right anterior superior temporal sulcus (raSTS) and in the right amygdala (Figure 2). These studies show how TMS-fMRI can map the functional connectivity of brain areas in humans that have been anatomically defined in non-human primates, and extend the cognitive inferences, based on TMS effects, beyond the site of stimulation.

A further advance is using TMS-fMRI to observe cognitive networks in the absence of task demands. Handwerker et al., (2019) carried out resting-state TMS-fMRI (rsfMRI) to map the remote effects of transient cortical disruption in the face network across the entire brain. TBS delivered over the rpSTS reduced resting-state connectivity across the extended face-processing network compared to TBS delivered over a control site. This connectivity reduction was observed not only between the rpSTS and other face-selective areas, but also between non-stimulated face-selective areas across the ventral, medial and lateral brain surfaces (e.g. right amygdala and bilateral fusiform face areas and occipital face areas). This result suggests that the typical functional connectivity of a network is dependent on all the normal operation of all the nodes in that network. The ability to observe network integrity in the absence of task demands makes TMS-rsFMRI of interest with clinical populations. For example, TBS combined with rsfMRI has been used to study the connectivity in aging (Abellaneda-Perez et al., 2019), visuospatial neglect (Fu et al., 2017), cerebellar connectivity (Rastogi et al., 2017) and depression (Baeken et al., 2017).

Combining rsfMRI with TMS can also be used to measure how stimulation can improve functional connectivity in an anatomical network. An impressive example of this approach is Wang et al., (2014) who investigated the cortical-hippocampal brain networks that underpin associative memory. They used rsfMRI to identify functional connectivity between the left hippocampus and the left parietal lobe. High frequency TMS $(20 \mathrm{~Hz}$ for 2 secs every 30 secs $)$ was then delivered over this left parietal site for 20 minutes over 5 consecutive days. TMS increased functional connectivity between the stimulation site and left hippocampus relative to a sham-TMS condition. This increase 
in functional connectivity was accompanied by improved behavioral performance on an associative memory task in which words were paired with faces. The increased connectivity between two brain areas that underlie associative memory, together with the associated behavioral improvement make this study compelling from both a scientific and a clinical perspective. From a scientific perspective, the anatomical specificity of the enhanced functional connectivity (replicated in Warren et al., 2019) demonstrates TMS can induce spatially distinct effects in anatomical networks. From the clinical perspective, the enhancement of memory has clear implications for potential treatments of patients with memory disorders.

Taking the TMS-fMRI advances together with the single-unit physiology findings we are clearly progressing towards an integrated understanding of the local, aerial, network and functional effects of TMS behaviour.

\section{Figure 2 about here}

\section{Spike Timing-Dependent Plasticity}

From the basic physiology of TMS effects and the network responses revealed by combinations of TMS-fMRI we learned that the inferences made about TMS in psychological experiments were vindicated in important ways. Great strides have also been made at the interface of physiology and behavior in studies using two coils to stimulate the cortex. This genuine advance, not even referenced in the "TMS Bible" (Wassserman et al, 2008) is spike timing-dependent plasticity (STDP) and refers to time-dependent changes in pre- and post-synaptic activity. This Hebbian mechanism had been studied in vitro and in vivo in small animals, and neuropharmacological studies have revealed the role of NMDA and Glutamate in the induction of Long Term Potentiation, and of GABAeregic synapses in inhibition (Caporale \& Dan, 2008) with remarkable temporal specificity. The requirement for Hebbian learning is that repeated paired stimulation of one area (Conditioning Pulse) followed by a second area (Test Pulse) a few msecs later increases synaptic efficiency between the two sites, resulting in the second area being more receptive to input from the first. Using TMS in humans, studies of the motor system have established that Paired Associative Stimulation (PAS), in which a peripheral nerve stimulus, paired with a cortical TMS pulse, could induce changes in MEPs if the two stimuli fell within a narrow time window of a few milliseconds. TMS over M1delivered 10ms after peripheral stimulation of the median nerve (MN) would reduce the subsequent MEP and an MN-M1interval of $25 \mathrm{~ms}$ would increase it (Müller-Dahlhaus et al., 
2010; Stefan et al., 2000, 2002; Wolters et al., 2003). These PAS studies were brought into the psychological domain by Rizzo et al., (2009) who showed that Hebbian plasticity could be induced by two coils placed over the cortex and measured both physiologically (MEP) and behaviorally. Rizzo et al called this ccPAS (cortico-cortical PAS) and we will use the term ccPAS to refer to all double cortex stimulation studies of plasticity ${ }^{1}$. It is a breakthrough study and contains all the components required to understand all other psychological ccPAS studies (Fig 3). Pairs of TMS pulses were delivered at $0.05 \mathrm{~Hz}$ for 30 minutes over M1 of the right hand (left hemisphere) and then M1 of the left hand (right hemisphere), with an 8msec delay between the hemispheres (left hemisphere M1 TMS is the conditioning stimulus here, and the right hemisphere M1 is the conditioned target). The intensity was set for each subject to produce a $1 \mathrm{mV}$ peak-to-peak EMG, which was between 115 and $125 \%$ of resting motor threshold. The physiological effect was to increase the MEP in the conditioned hand representation and to decrease interhemispheric inhibition (IHI). IHI was measured by delivering single pulses over left and right M1 with long delays of 35-45 msecs that are known to produce inhibition of the MEP response (Daskalakis, 2004). Behaviourally the ccPAS speeded simple reaction time responses with the left index finger (the conditioned finger representation) for up to 30 minutes post ccPAS. This experiment is a watershed for ccPAS in TMS studies of behaviour and plasticity. After 70 years of knowing the Hebb rule, 100 years of learning research, and 35 years of TMS, psychologists now have a method for studying Hebbian plasticity in learning with human brain stimulation.

\section{Figure 3 about here}

The M1 effects in ccPAS seem to be robust and replicable, but since Rizzo et al.,(2009) behavioral change has been more elusive. Buch et al., (2011) conditioned M1 with ventral premotor (PMv) TMS while subjects performed a reach and grasp task. The STDP effect, which lasted for at least an hour, was state-dependent (see later section State-Dependency) in that when the subject was performing the task the PMv TMS induced excitation over M1, but when at rest PMv TMS increased inhibitory effects of PMv on M1. There was no effect on the performance of the task itself, however. Chao et al., (2015) also obtained a ccPAS change in M1 excitability by conditioning

${ }^{1}$ The terminology in the literature is not friendly to the newcomer. Experiments using the same method are variously referred to as PAS, cPAS (meaning cortical PAS), or ccPAS (meaning corticocortical PAS) or simply as paired stimlation. Here we use PAS to refer only to studies in which peripheral nerve stimulation is paired with M1 stimulation. For studies in which two cortical sites are stimulated we use the term ccPAS after Rizzo et al., 2009. 
with TMS over posterior parietal cortex 8 msecs prior to M1. Like Buch et al., the STDP effects on the MEP lasted over an hour but they had no effect on a peg board task. Johnen et al., (2015) used ccPAS to examine whether inducing a stronger connection between PMv and M1 (90 pulse pairs at $0.1 \mathrm{~Hz}$ with a PMv-M1 ISI of 8ms) would translate to an increase in functional connectivity measured by fMRI while subjects performed a reach and grasp task. Here, ccPAS increased the strength of connectivity between the two areas during the performance of the task, but not at rest. Subsequent analysis showed that the PMv-M1 ccPAS had specific effects on interactions between other nodes in the sensorimotor network. Functional connectivity was increased between PMv and AIP, and decreased between PMd and M1 and between PMd and pSPL. There were no effects on behaviour measures.

Santarnecchi et al (2015) also examined how ccPAS changes cortical network activity. They applied ccPAS over a parietal region of default mode network (DMN) and a region of the prefrontal cortex in the task-positive network (TPN) (Raichle, 2015). The two regions they stimulated were negatively correlated nodes of their respective networks, identified by individual fMRI. Following a previous study of frontoparietal STDP they adopted three temporal conditions: DMN/TPN ISI $+10 \mathrm{~ms}$, DMN/TPN ISI -10ms, DMN/TPN ISI 0ms. Pairs of pulses were delivered at $0.2 \mathrm{~Hz}$ over 15 mins. Rather than measuring STDP in motor cortex, they asked how different parts of cognitive networks respond to TMS test pulses when the dependent variable is the BOLD signal.

\section{Figure 4 about here}

Two findings of major interest here are when the DMN pulse preceded the TPN pulse by $10 \mathrm{~ms}$ there was a clear change in the negative correlation of the two sites, and in a third of subjects it became positive (Fig 4). The effects, like those of Buch et al., (2011) were state-dependent. If the correlated activity between the two sites was weaker prior to ccPAS, the effects consistent with STDP were increased in size. There was no report of any behavioral effects.

Part of the interest in ccPAS is the hope that STDP may prove clinically useful, but most of the studies post-Rizzo et al., (2009) either do not measure or do not see a behavioral change consequent on the STDP despite significant physiological changes. Another attempt to match the physiology of ccPAS-induced STDP to behavior was made by Kohl et al., (2019) who bi-directionally stimulated inferior frontal cortex (IFC) and pre SMA with ISIs of either $4 \mathrm{~ms}$ or $10 \mathrm{~ms}$. Based on Weise et al's (2013) PAS model they hypothesised that stimulating the pre-SMA 4 or 10ms prior to IFC would inhibit the subthalamic nucleus (STN) and interfere with a behavioral task, and that 
stimulating IFC 4 or $10 \mathrm{~ms}$ prior to pre SMA would facilitate the STN and task performance (see Fig 2 of Weise et al., 2013). They delivered 100 pairs of ccPAS pulses at $0.2 \mathrm{~Hz}$. The two tasks they gave to their subjects were the Stop Signal Reaction Time Task (SSRT) and a temporal discounting task. While they reported some effects on a stop signal RT test, there were no effects on a delay discounting task.

The clear challenge in this area is to bridge the gap between the physiologically robust STDP effects (measured by MEPs or fMRI) and behavior. Two studies in particular may help to direct the next steps: Veniero et al., (2013), and Momi et al., (2019). They each raise different issues to be pursued from physiological, EEG, imaging, and behavioral perspectives.

Veniero et al., induced LTP- and LTD-like STDP and measured the effects on evoked potentials. They induced bi-directional STDP by stimulating PPC and M1 with 100 pulse pairs over 8 minutes with an ISI of 5 msecs (see also Koch et al., 2013). When ccPAS had an inhibitory effect on the MEP there was an increase in beta coherence between PPC and M1. When ccPAS induced an excitatory effect as measured by the MEP there was an increase in alpha coherence between the two regions. Given the detailed work on TMS-EEG in vision, attention, and awareness in particular (Herring et al., 2015; Romei et al., 2008; Taylor, 2018), it is clear that Veniero et al's paradigm is both possible and worthy of extension. The combination of ccPAS with EEG may help to understand why the physiological effects do not have behavioral consequences as often as one might hope.

The second issue for replication and development is that of producing psychological effects. Momi et al., (2019) used ccPAS to try to synchronize two positively correlated nodes of the left fronto-parietal network. They tested ccPAS bi-directionally with pairs of pulses delivered for 15 mins at $2 \mathrm{~Hz}$ with an ISI of $10 \mathrm{~ms}$. Over 5 weeks of repeated stimulation and behavioral testing they observed STDP and indications of directional-specific behavioral changes. Fronto-Parietal ccPAS was associated with a small improvement in relational reasoning, and Parieto-Frontal ccPAS with an improvement on a logical reasoning task (see Fig 3 of Momi et al., 2019). This is a rare example of ccPAS producing a complex behavioral change. We will return to this issue in our survey of StateDependency below hope (see also Chiappini et al., 2018; Nord et al., 2019; Romei et al., 2016a).

\section{State-Dependency}

In one sense, all TMS research is based on state-dependency. Comparison of a resting and active MTs is a measure of state-dependent motor thresholds. There have been many examples of behavioral context affecting TMS in the motor system (Bestmann et al., 2008a; Bestmann \& Krakauer, 2015). All of these examples were demonstrated by measuring an effect on the MEP, but 
for state- dependency to be useful in studying psychological concepts not accessible to the MEP we needed a new approach. The state-dependent approach in perception and cognition experiments is owed mostly to the efforts of Juha Silvanto. The effects of TMS are dependent on the current state of excitation of the brain tissue being stimulated. In cognitive experiments, however, we have no direct measure, such as the MEP, of the current state of, say, the PPC, FEF, DLPC, OFA etc. Silvanto's major contribution was to leverage state-dependency in psychological experiments to enhance the functional resolution of TMS to selectively target specific, even overlapping neural representations.

Silvanto used adaptation to influence the initial state of the region being stimulated. His first study (Silvanto et al, 2007) is simple, elegant, and serves as a model for thinking about statedependent TMS in cognition. Subjects adapted to colour/orientation stimuli for 30 secs and were required to report the colour of a test stimulus. TMS was delivered at different times after presentation of the test stimuli. On non-TMS trials subjects experienced the complementary colour of the adapting stimulus, but TMS over the visual cortex induced a bias towards the original, adapted stimulus colour.

The method has now been used widely in studies of mental imagery (Cattaneo et al., 2012), short-term memory (Silvanto and Soto, 2012), letter selection, abstraction, action observation, and semantic processing (Cattaneo et al., 2008, 2009, 2010). Cattaneo et al., (2010) is of particular interest because it observed a naming effect with TMS delivered outside visual or language areas. They tested the effects of TMS on category-specific neuronal representations in the encoding of tool words in the left ventral premotor cortex (PMv). Subjects were primed with a category name ("tool" or "animal") to adapt the PMv to one or other category of objects. TMS was then applied at the onset of a target word that was either congruent or incongruent with the primed category. The outcome was the removal of the priming effect.

Another example of non-sensory effects of state-dependent TMS comes from a study of action recognition. Mazzoni et al., (2017) adapted subjects to point-light displays of affective movements. They applied rTMS at the onset of the point-light displays, over the aIPS, pSTS, or occipital pole. Only TMS over aIPS had any effect on subjects' identification of the happy or fearful motion displayed, and it served to reverse the effects of adaptation.

We can now bring the two recent advances of spike timing-dependent plasticity, and statedependent TMS together. As we noted in the introduction, and we shall see again in Behavioral Findings below, there is increasing integration of TMS with other methodologies. Three studies serve as useful examples of how state-dependency and ccPAS may be used in conjunction. The first of these studies (Arai et al., 2011) used three coils to stimulate M1 bilaterally and SMA using ccPAS . 
They induced bidirectional and spatio-temporal-specific STDP, enhancing the MEP if the SMA-M1 delay was $6 \mathrm{~ms}$, and decreasing it if the M1-SMA delay was $15 \mathrm{~ms}$. The new factor here was the requirement that for these associations to occur, both left and right M1 had to be stimulated perisimultaneously. It's a complex experiment, but the pattern of advances in TMS and cognition to date has followed the path of finding the most interesting advances in motor physiology and adapting them for our own purposes. It may be that the next phase of state-dependent, Hebbian plasticity in psychological studies will require three coils.

The second study Romei et al (2016a) is important for being an example of ccPAS outside the motor system. Using V5 and V1 stimulation, at $0.1 \mathrm{~Hz}$ for 90 pairs of stimuli with an ISI of $20 \mathrm{~ms}$, they improved subjects' performance on a motion coherence task. In a follow up study Chiappini et al, (2018) showed that this strengthening of re-entrant V5-V1 projections was state-dependent (Fig 5). Subjects were adapted to a direction of motion during which ccPAS was delivered. In other words this is a state-dependent ccPAS protocol. Based on previous experiments V5 was stimulated at $80 \%$ of phosphine threshold and V1 at phosphene threshold. Controls were administered for time (a V5-V1 delay of 100ms) and direction (V1 preceding V5). The outcome was that when tested on a direction of motion threshold detection task, sensitivity was increased in the direction congruent with the ccPAS stimulation. As the authors put it, this "allows targeting of cortico-cortical pathways associated with specific functions."

\section{Figure 5 about here}

\section{Behavioral Findings}

Many inventive and impressive behavioral experiments have exploited advances in our understanding of psychological processes and combinations of TMS with other methods. Some of the studies discussed in previous sections were chosen because they provided the first and/or good illustrations of TMS-imaging, STDP, or state-dependency. This section surveys the breadth of TMSbehavioral effects.

Visual awareness has been the subject of many TMS studies. Allen et al., (2014) used offline cTBS to reproduce blindsight-like effects. As we saw in the Physiology and Network sections, theta stimulation may not have a single effect at a single site. By combining cTBS with magnetic resonance spectography (MRS) and magneto-encephalography (MEG) Allen et al., were able to show that although cTBS reduced cortical activity and increased GABA concentrations, awareness 
thresholds were decreased rather than increased as predicted. The behavioral and MRS effects of cTBS were stable between 0 and 48 mins post cTBS so it is not possible to assess the results with Romero et al., (2019a) in mind, but they provide a good example of how physiologically inhibitory TMS can result in behavioral enhancements. The same group also used MEG as a chronometric guide to use TMS to test the roles of preSMA and inferior frontal cortex in response inhibition (see Allen et al., 2018).

A related example of improvement following offline stimulation shows how using psychophysical tasks can help to parse possible mechanisms. Tadin et al., (2011) used 1Hz TMS for 15 minutes over V5 or posterior occipital cortex and gave subjects a motion discrimination task with stimuli of different sizes. TMS over V5 is usually used to disrupt motion perception, but the area is also involved in spatial suppression because of its large receptive fields. Tadin et al., therefore used the psychophysical manipulation to show that disruption of the suppression caused by V5 TMS led to an improvement on movement discrimination with large visual stimuli. So we see a pattern emerging in TMS and enhancements: TMS, which is still largely thought of as a "neurodisruptive" method, can elicit functional improvements due to state-dependency (e.g. Silvanto et al., 2007), psychophysical parameters (e.g. Tadin et al., 2011), different phases of physiological effects (e.g. Romero et al., 2017, 2019a,b), or inhibition of connected areas (e.g. Allen et al., 2014).

Attentional functions in the parietal cortex have long been a focus of TMS research (e.g. Herring et al., 2015; Mahayana et al., 2014; Olk et al., 2015) but behavioral studies within the last ten years have revealed attentional affects in other areas (Rangelov et al., 2015), anatomical specificities for other functions in human parietal cortex (Crawford et al., 2011; Vesia et al., 2010), and interactions between attention and TMS physiological effects (Bergmann et al., 2016; Herring et al., 2015; Romei et al., 2016b; Taylor, 2018; Thut et al., 2017). Where Allen et al., (2007) asked whether changes in occipital excitability would change visual awareness, Rangelov et al., asked whether feedforward retinotectal input is necessary for attentional capture to occur. They bypassed retinotectal input by inducing phosphenes with TMS as valid and invalid cues. The phosphenes produced similar, though slightly weaker cueing effects than real visual stimuli, but the paradigm of bypassing anatomical pathways by use of phosphenes is one that can be generalized and used in TMS-fMRI, TMS/EEG, and state-dependent experiments. Staying with attentional functions, Herring et al., (2015) showed that single-pulse TMS-induced oscillatory activity in the visual system has the same neural origins and character as endogenous oscillations (thanks to Romero et al., we can now begin to look at those origins with physiological specificity). They measured transcranial evoked potentials (TEP) and EEG alpha responses while subjects attended to 
either a visual or auditory stimulus in a cross-modal attention task. An early component of the TEP (N40) was enhanced by TMS while TMS-locked alpha power was reduced during visual attention conditions. It's tempting to note the similarity of timing between the TEP and the single units in Romero et al., but we would caution against expecting single units, EEG, and behavioral chronometric to show the same temporal patterns because they measure different neuronal events (see Fig 5 of Walsh \& Cowey, 2000).

The parietal cortex is perhaps over dominated by attention research in psychology so it is important to remind ourselves that it has other functions. Vesia et al., (2010) and subsequent work from the same group have elegantly parsed the functional specificity of human parietal areas based on single unit evidence from non-human primate studies (Fig 6). They delivered triple pulses of $10 \mathrm{~Hz}$ TMS over superior parietal occipital cortex (SPOC), and two sites in intraparietal sulcus (mid mIPS, and more caudally over the angular gyrus AG). Subjects were cued to perform a delayed spatial match saccade or reach task and the rTMS was delivered during the delay period. TMS over mIPS and AG both increased the variability of reaching responses and impaired reach and saccade accuracy for contralateral targets. TMS of SPOC impaired reach accuracy but did not affect saccades.

\section{Figure 6 about here}

We will end with an area of investigation that exemplifies everything we have highlighted in this review: memory. Traditionally one might begin at the end, so to speak, in the hippocampus or the frontal lobes, but there have been remarkable memory-related findings from TMS studies of occipital lobe function. Hilbert et al., (2019) described what one might call "trait-dependent" TMS effects. By administering digit span tasks to subjects and taking subjective reports of their mnemonic strategies, Hilbert et al., discovered that $1 \mathrm{~Hz}$ TMS, close to the occipital pole, given offline for 10 mins before the task impaired memory performance in subjects who were categorised as visualisers. This shows that TMS is useful in identifying inter-individual differences in working memory processes. Several TMS studies have indicated a role for sensory cortex in working memory (Campana et al., 2006; van de Ven \& Sack, 2013). Zokaei et al., (2014), for example, using online rTMS over V5, established that the probability of remembering the direction of motion of an object was a function of task-relevance and serial position, not parameters one habitually associates with sensory cortex. Their result may appear counter-intuitive in that more recent stimuli were disrupted while more distant items were facilitated by TMS over V5. A related effect was obtained with single pulse TMS over occipital cortex by van Lamsweerde \& Johnson (2017) 
who disrupted the number of items subjects could remember. The timing of the TMS effects of both these experiments falls within the first two phases of the single unit responses discussed in our Physiology and Behavior section.

The behavioural experiments we have reviewed testify to the use of TMS in addressing psychological theories, in this case the sensory recruitment model of working memory (D'Esposito \& Postle, 2015), but they are part of something more. There is an important conceptual and mechanistic link to be made here, and it speaks to a common physiological principle of TMS that lives in different corners of our literature under different guises for no better reason than we are interested in different psychological phenomena. In the state-dependent experiments we see TMS activating adapted neuronal populations (Silvanto, 2007); in the working memory experiments we see TMS preferentially activating temporally "less-privileged" representations (Zokaei et al., 2014); in phosphene awareness experiments we see TMS reactivating weak "echoes" (Jolij \& Lamme, 2010); and in the working memory reactivation experiments (Rose et al., 2016) we see TMS preferentially activating a currently (but to-be) relevant population. So, across physical parameter space (Silvanto), time (Zokaei), relevance (Rose), and state of awareness (Jolij \& Lamme) there is a common picture of TMS preferentially stimulating weakly activated populations. We began this review by emphasising the need to understand the physiology of TMS better, but intellectual is never one way, and these behavioural experiments set new questions for future physiological recordings. Where we were asked how we know what TMS is doing, we can now return the question to the electrophysiologists with psychological dividends and ask "what do our psychological states do to the physiological responses?"

\section{Conclusions}

So where are we now? We may be accused of being over-optimistic about the advances in the last 10-12 years, but we'd enter a plea of not guilty. The foundations such as STDP, state-dependency, anatomical reliability, MEPs, phosphenes, and network effects have depth in a literature that is almost unrecognisable from the "point and shoot" era predating our review. It is true that sample sizes are often small or appear so compared with pure behavioral experiments (combining TMS with fMRI/EEG/MEG/MRS, eye movements/psychophysics/MEP, and using two coils isn't easy), and effect sizes go unreported (or selectively so). There have been few pre-registered studies, and even some of those few have based much of their interpretation on unplanned analyses. All of these are weaknesses and are fixable: It is important to report null results (de Graaf \& Sack, 2011); consider pre-registration of experiments (Nosek et al., 2018); adequately report the stimulation 
methods and dosage (Peterchev et al., 2012); adopt and adapt open data practices from other areas of research (Nichols et al., 2017). However, these issues are neither unique nor even particularly salient in our field compared to others, and slow and clumsy though the progress may seem to some, slow and clumsy are not entirely bad ways of making scientific progress (Frith, 2020; Lewandowsky \& Oberauer, 2020). We encourage all authors and reviewers to partake in improving the overall quality of the field. However, we do not want to diminish the significance of the efforts, results, and knowledge that have accrued, and the overall message from the literature surveyed is overwhelmingly positive. The field has moved on technically (e.g. Allen et al., 2014, 2018; Polanía et al., 2018; Thut et al., 2017), conceptually (Romero et al., 2017, 2019a,b; Chiappini et al., 2018), and empirically (e.g. Amemiya et al., 2017; Brown et al., 2019a,b; Ruzzoli \& Soto-Faraco, 2014;

Willacker et al., 2019). If, as Medawar states, "research is surely the art of the soluble" (1967, p 87), the last decade or so of research in TMS and cognition has, at the very least, brought many questions into that realm of the soluble. 
Acknowledgements: We are grateful to Ethan Buch, Roi Cohen Kadosh, Marco Davare, Eva

Feredoes, Peter Janssen, Carlo Miniussi, Uinsionn O'Breathnach, Beth Parkin, Angelo Quartarone,

Vincenzo Romei, Matthew Rushworth, Emiliano Santarnecchi, Hartwig Siebner, Juha Silvanto,

Angela Vaughn, and Michael Vesia for comments, assistance and guidance on different parts of this review. 


\section{Figure Legends}

Figure 2. The results of Pitcher et al. (2017). Percent signal change data for the dynamic face, body and object stimuli before and after TBS in the five core regions-of-interest (ROIs): the rpSTS, raSTS, right amygdala, rFFA, and rOFA (Error bars denote Standard Errors). Brain slices show examples of the face-selective (dynamic faces > dynamic objects) ROIs in a typical participant. TBS delivered over the rpSTS selectively and significantly reduced the BOLD response to dynamic faces only in the rpSTS, raSTS and right amygdala (* denotes significant effects). 